\title{
Robert William Smith (1807-1873): Patólogo y Cirujano, la historia detrás de la fractura, de la fractura de Colles invertida
}

\author{
Robert William Smith (1807-1873): Pathologist and Surgeon, the \\ Story behind the fracture, on the reverse Colles fracture
}

Leopoldo Mario Aguayo González*

*Catedrático e Investigador en Historia de la Medicina. Profesor de Músculo Esquelético en la Universidad Latina de México, Celaya, Guanajuato. Profesor de Biomecánica, Prótesis y Ortesis, Ingeniería Biomédica, Universidad de Celaya. Profesor e Investigador en Historia de la Ortopedia y Traumatología. Federación Mexicana de Colegios de Ortopedia y Traumatología A. C. (FEMECOT). Sociedad Latinoamericana de Ortopedia y Traumatología Infantil. (SLAOTI). Académico de la Sociedad Potosina de Estudios Médicos AC. (SPEM). Titular del Comité de Historia y Arte de la (FEMECOT), Mesa Directiva $2020-2022$.

\section{Resumen}

Robert William Smith es mejor conocido por su descripción de lo que hoy conocemos como la fractura de Smith o de Colles invertida, fractura por supinación del radio distal. Sin embargo, como muchos médicos notables cuyos nombres están unidos a ciertas condiciones médicas y quirúrgicas, su contribución a la medicina fue mucho mayor que la sola descripción de una entidad específica. Llama la atención que conociendo el trabajo previo publicado póstumamente en 1783 de Claude Pouteau sobre la fractura por pronación del radio distal, y siendo Robert William Smith un consumado lingüista, no la designase con ese nombre; en la literatura francesa esta fractura es conocida como fractura de Pouteau-Colles. En 1847 publicó su libro A Treatise on Fractures in the Vicinity of Joint and on Certain Forms of Accidental and Congenital Dislocations. Dublín: Hodges \& Smith. Robert William Smith al editar su libro en 1847 anotó: «Es, creo, el deber de toda persona que se compromete a escribir sobre un tema determinado, familiarizarse en la medida de lo posible y reconocer el trabajo de aquéllos que pueden haberlo precedido en el mismo campo". Durante su tiempo, RW Smith fue un reconocido médico. Incluso al otro lado del Océano Atlántico desde su Irlanda natal la Revista Americana de las Ciencias Médicas escribió que su tratado sobre fracturas «puede considerarse uno de nuestros mejores modelos para realizar observaciones quirúrgicas". Escribió también en 1849 sobre la enfermedad hoy conocida como neurofibromatosis (tipo I), 33 años antes de que Friedrich Daniel von Recklinghausen lo publicara en 1882. Trabajó junto a gigantes de la medicina como Abraham Colles, Robert Adams, William Stokes, Robert James Graves y Dominic Corrigan, todos ellos médicos irlandeses.

Palabras clave: Historia de la medicina, de la traumatología y de las fracturas.

\section{Abstract}

Robert William Smith is best known for his description of what is now known as the Inverted Smith or Colles fracture, a supination fracture of the distal radius. However, like many notable physicians, whose names are linked to some medical and surgical conditions, his contribution to medicine was far greater than the single description of a specific entity. It is striking that knowing the previous work published posthumously in 1783 by Claude Pouteau on the pronation fracture of the distal radius, since Robert William Smith was an accomplished linguist, he did not designate it by that name; In French literature this fracture is known as the Pouteau-Colles fracture. In 1847, he published his book «A Treatise on Fractures in the Vicinity of Joint and on Certain Forms of Accidental and Congenital Dislocations». Dublin: Hodges \& Smith. Robert William Smith when editing his 1847 book scored. «It is, I believe, the duty of every person who undertakes to write on a particular subject, familiarize himself, as far as possible, and acknowledge the work of those who may have preceded him in the same field". During his time, RW Smith was a renowned physician. Even across the Atlantic Ocean from his native Ireland, the American Journal of Medical Sciences wrote that his treatise on fractures «may be considered one of our best models for making surgical observations". He also wrote in 1849 about the disease today known as neurofibromatosis (type I), 33 years before Friedrich Daniel von Recklinghausen published it in 1882. He worked alongside medical giants like Abraham Colles, Robert Adams, William Stokes, Robert James Graves, and Dominic Corrigan, all of them Irish physicians.

Keywords: History of medicine, history of traumatology and fractures.

Correspondencia:

Dr. Leopoldo Mario Aguayo González

E-mail: aguayoleopoldo@gmail.com

Recibido: 03-02-2021. Aceptado: 07-02-2021.

Citar como: Aguayo GLM. Robert William Smith (1807-1873): Patólogo y Cirujano, la historia detrás de la fractura, de la fractura de Colles invertida. Orthotips. 2021; 17 (1): 55-58. https://dx.doi.org/10.35366/99168 


\section{Introducción}

Robert William Smith es mejor conocido por su descripción de lo que hoy conocemos como la fractura de Smith. Sin embargo, como muchos médicos notables cuyos nombres están unidos a algunas condiciones médicas y quirúrgicas, su contribución a la medicina fue mucho mayor que la sola descripción de una entidad específica.

Escribió también sobre la enfermedad hoy conocida como neurofibromatosis antes que Von Recklinghausen lo publicara. ${ }^{1}$

Durante su tiempo, RW Smith fue un reconocido médico. Incluso al otro lado del Océano Atlántico desde su Irlanda natal, la Revista Americana de las Ciencias Médicas escribió que su tratado sobre fracturas «puede considerarse uno de nuestros mejores modelos para realizar observaciones quirúrgicas».

Trabajó junto a gigantes de la medicina como Abraham Colles, Robert Adams, William Stokes, Robert James Graves y Dominic Corrigan, todos ellos médicos irlandeses. ${ }^{2}$

\section{La vida de RW Smith}

Robert William Smith nació el 12 de octubre de 1807 en Dublín, aprendiz de Robert Carmichel (17761849), inició estudios en la Medicine School of the Royal College of Surgeons of Ireland, Trinity College, se licenció en medicina en 1832, obtuvo su doctorado en 1842 y fue miembro del RCSI (Royal College of Surgeons of Ireland) en 1844 (Figura 1).

Su reputación como patólogo era tan grande que cuando Abraham Colles (1773-1843), retirado del servicio activo desde 1836, enfermó de gota y falla cardiaca, solicitó al mismo RW Smith en 1842 que realizara su autopsia y determinara la causa de su muerte. Los hallazgos: pulmón izquierdo fibrótico y dilatación grasa cardiaca fueron publicados por William Stokes en diciembre de 1843, Observations on the Case of the Late Abraham Colles, M.D., formerly Professor of Surgery of the Royal College of Surgeons in Ireland. W Stokes fue secretario conjunto de la Sociedad Patológica de Dublín con RW Smith por 35 años. ${ }^{3}$

Prominente médico en Irlanda, excelente lingüista, historiador médico y patólogo quirúrgico publicó temas que van desde la artritis hasta el neuroma.

A partir de 1843 fue cirujano del Dispensario General Talbot, del Asilo para Lunáticos en la Isla
Bridge y del Hospital y Escuela de Medicina Richmond, y fue nombrado profesor de cirugía en el Trinity College en 1847.

Enseñó cirugía clínica y medicina forense en Richmond durante muchos años. El excelente museo del hospital fue posible gracias a sus esfuerzos.

Su pasión por la patología lo inspiró a fundar en 1838 la Sociedad Patológica de Dublín, donde se desempeñó como su secretario por 35 años y donde tuvo una excelente oportunidad para reunir múltiples preparaciones patológicas. Era vicepresidente del Royal College of Surgeons of Dublin cuando ocurrió su muerte el 28 de octubre de $1873 .^{3}$

\section{Fractura del radio}

En 1847 publicó su libro $\boldsymbol{A}$ Treatise on Fractures in the Vicinity of Joint and on Certain Forms of Accidental and Congenital Dislocations. Dublín: Hodges \& Smith. $1847 .{ }^{4}$

Es el primer trabajo importante sobre fracturas de un autor irlandés. Incluye la descripción de la fractura que lleva su nombre (Figura 2).

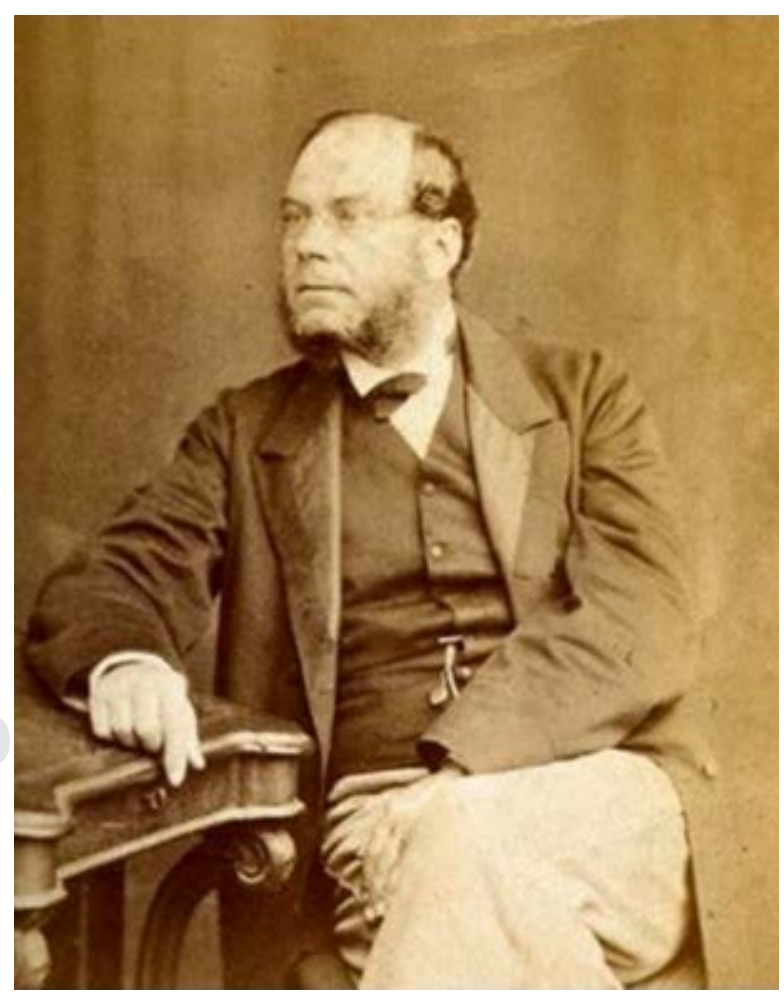

Figura 1: Robert William Smith (1807-1873). 


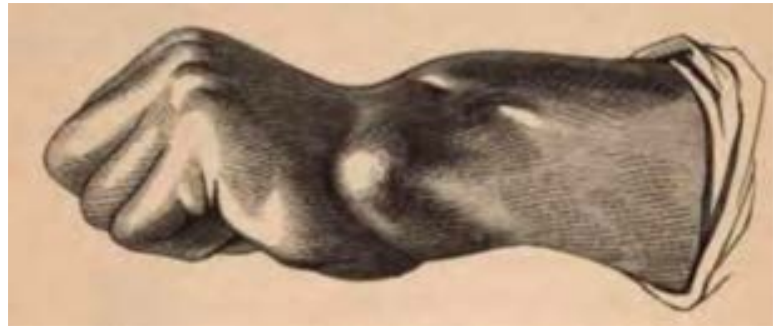

Figura 2: Fractura de Smith, dibujo del autor $1847 ., 5$

Antes que Smith, otro tratado inglés que hablaba sobre dislocaciones y fracturas, fue publicado por Astley Paston Cooper (1768-1841) en 1822.

En el capítulo "Sobre fracturas de los huesos del antebrazo en las proximidades de la articulación de la muñeca» Smith corrigió la descripción original de Colles «This fracture takes place at about an inch and a half above the carpal extremity of the radius" que había localizado el sitio de su fractura más distalmente, pues tuvo la oportunidad de realizar autopsias a 20 pacientes que habían presentado estas fracturas. ${ }^{3-5}$

En sus propias palabras señaló: "The situation of the fracture is not so high as Mr. Colles states it to be; I have never seen it more than an inch above the carpal end of the bone; in the majority of cases it is not so much». Añadió: «En este país el nombre de un eminente cirujano, recientemente fallecido, se ha asociado con esta lesión, la que actualmente conocemos como fractura del radio de Colles». . $^{3-5}$

Esto es interesante de señalar porque Abraham Colles de hecho no fue el primer cirujano en describir el desplazamiento dorsal en la fractura del radio. Aunque A. Colles no estaba enterado del trabajo previo de Pouteau, RW Smith sí lo conocía. Esta fractura descrita inicialmente por Claude Pouteau (1724-1775) de Lyon, Francia fue publicada póstumamente en 1783. ${ }^{4}$ Antes de que Pouteau escribiera sobre las fracturas de la muñeca, era generalmente aceptado que estas lesiones eran luxaciones de la muñeca y no fracturas.

Respetuosamente RW Smith escribió que el error anatómico de Colles se debía a que éste nunca realizó una autopsia a ninguno de sus pacientes con esa fractura. ${ }^{3-5}$

RW Smith unió firmemente el epónimo de Colles a la fractura más común de la muñeca que éste describió y que apareció en un artículo en la revista Edinburgh Medical and Surgical Journal; 1814 Apr 1; 10 (38): 182-186. ${ }^{3-6}$
Y utilizando sus propias palabras para describir la fractura que actualmente se asocia con su nombre, RW Smith anotó: «Ésta es una lesión de ocurrencia extremadamente rara que presenta características muy parecidas a las de la dislocación del carpo hacia adelante"..$^{3-5}$

Hay que tomar en cuenta que el diagnóstico de las lesiones óseas se hacía sólo sobre bases clínicas, faltaban aún 48 años para que se realizara el descubrimiento de los rayos X en 1895 por Wilhelm Conrad Rontgen (1845-1923) de Wurzburgo, Alemania.

Escribió: "Generalmente esta fractura ocurre como consecuencia de una caída sobre el dorso de la mano, y se ubica de media a una pulgada por encima de la articulación».3-5

Con base en dicho caso se hizo el dibujo anterior, el paciente, en un esfuerzo por evitar ser atropellado por un auto cayó con gran violencia sobre la parte trasera de su muñeca; el extremo inferior del radio estaba roto y desplazado hacia adelante junto con el carpo.,

Sin embargo, la fractura de Smith, tal como la conocemos hoy, fue descrita por otro cirujano francés de Aix-en-Provence, Jean Gaspard Blaise Goyrand (1803-1866): Mémoires sur les fractures

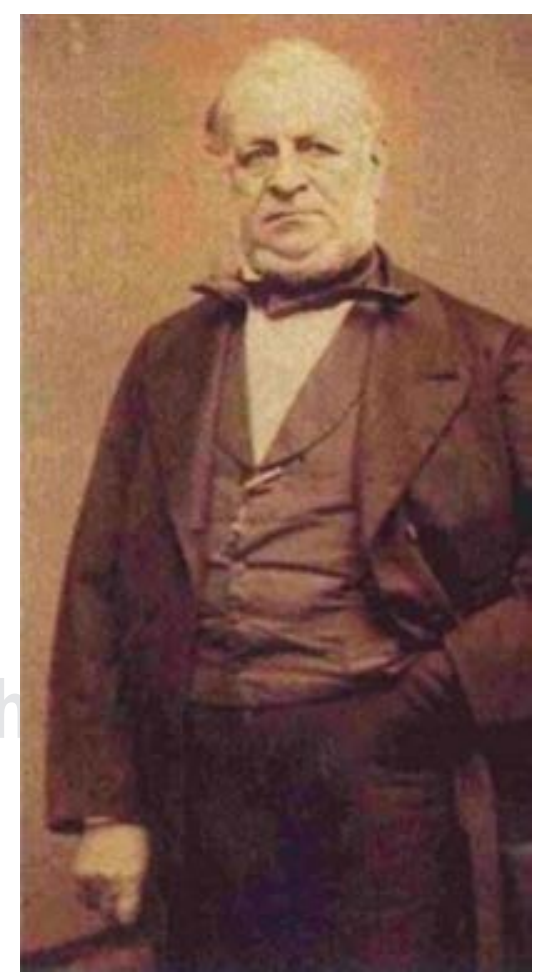

Figura 3: Jean Gaspard Blaise Goyrand (1803-1866). 
de l'extrémité inférieure de radius, qui simulent les luxations du poignet. Gazette médicale de $\mathrm{Pa}$ ris. 1832; 3: 664-6673 (Figura 3). La descripción que hizo RW Smith de esta fractura era más detallada, aunado al hecho de que era una figura prominente en la medicina mundial del siglo XIX.

Sir Charles Alexander Cameron (1830-1921) en su Historia de la RCSI de 1886, afirma que RW Smith fue uno de los anatomistas y cirujanos más distinguidos de su tiempo que haya producido Irlanda, y como profesor rara vez ha sido igualado y probablemente nunca superado. ${ }^{7}$

\section{Otras publicaciones}

1. On the diagnosis of fractures of the neck of the femur with plate. Published 1834.

2. An essay upon the original or congenital luxations of the upper extremity of the humerus. Published in (1839).

3. Observations on the diagnosis and pathology of fractures of the neck of the femur. Published in (1840).

4. A treatise on the pathology, diagnosis, and treatment of neuroma. Dublin, Hodges and Smith. Published in (1849). Smith differentiated patients with traumatic neuromas from those with cases of multiple, idiopathic neuromas.

Este libro incluye una descripción completa de la neurofibromatosis (tipo I) 33 años antes de que Friedrich Daniel von Recklinghausen (18331910) de Estrasburgo, Alemania la describiera y popularizara el término en 1882. Ese mismo año fue nombrado primer profesor de cirugía en el Trinity College de Dublín. 4,5

\section{Más Publicaciones}

1. Observations on disjunction of the lower epiphysis of the humerus. Published in (1850).
2. Observations upon chronic rheumatic arthritis of the shoulder. Published in (1853).

Termino este artículo anotando lo que RW Smith escribió al editar su libro en 1847: ${ }^{3}$

Es, creo, el deber de toda persona que se compromete a escribir sobre un tema determinado, familiarizarse en la medida de lo posible y reconocer el trabajo de aquéllos que pueden haberlo precedido en el mismo campo. ${ }^{3-5}$

Falleció de enfermedad hepática acompañada de ascitis. Su puesto de profesor de cirugía en el Trinity College de Dublin fue ocupado por Edward Hallaran Bennett (1837-1907), otro gigante de la ortopedia irlandesa que describió su epónimo en 1882 sobre la fractura de la base del primer metacarpiano del pulgar. ${ }^{4}$

\section{Referencias}

1. Peltier LF. Fractures: a history and iconography of their treatment, Norman Publishing, San Francisco CA, Second Printing 1999, pp. 42-44.

2. Rang M. The story of orthopaedics, W.B. Saunders Company, Philadelphia PA, Third Edition, 2000, pp 234-235, 405-406.

3. Shah HM, Chung KC. Robert William Smith: his life and his contributions to medicine. J Hand Surg Am. 2008; 33 (6): 948-951.

4. Smith RW. A treatise on fractures in the vicinity of joints, and on certain forms of accidental and congenital dislocations. Dublin, Hodges and Smith, 1847.

5. Lee TC. A trinity of Eponymus surgeons II-Robert William Smith (1807-1873) \& Smith's fracture of the radius (1847) University of Pennsylvania Orthopaedic Journal. 2005-2006; 18: 89-91.

6. Colles A. On the fracture of the carpal extremity of the radius. Published in the Edinburgh Medical and Surgical Journal. 1814; 10 (38): 182-186.

7. Cameron CA. History of the Royal College of Surgeons in Ireland (RCSI) and of the Irish schools of Medicine including Numerous Biographical Sketches also a Medical Bibliography. Dublin: Fannin \& Company; 1886, p. 810. 